\title{
Refractivity Measurements on Thick Plates
}

\author{
James B. Saunders
}

\begin{abstract}
An interferometer is described that permits measurement on refractive gradients in thick transparent bodies. The order of interference does not depend upon the thickness of the specimen. Any monochromatic light source may be used to test plates of any thickness. A 10-inch aperture interferometer of this type has been constructed and used successfully to measure index gradients in thick glass disks.
\end{abstract}

\section{Introduction}

The increasing use of large optical elements, such as wind-tunnel windows, large aerial-camera lenses, and large interferometers, has increased the need for high-precision testing of optical homogeneity in thick glass plates. Interferometric precision is essential for windows that are to be used in interferometric wind-tunnel work. The requirements of coherence limits the thickness of plates that may be measured by interferometers that have previously been used for index measurements. An interferometer arrangement for measuring optical homogeneity of glass plates, and which is not limited by optical-path differences due to plate thickness, is described. It has greater stability and freedom from vibrations than other interferometers used for this purpose, but the interpretation and computation of results are somewhat more intricate than that for the Twyman-Green type, for instance.

\section{Optics of the Interferometer}

The optical arrangement of this interferometer is shown in figure 1. The assembly is a modification of the Dowell angle-gage interferometer ${ }^{1}$ shown in figure 2. This modification permits larger working space than is available with the Dowell arrangement, which is essential for measuring large specimens without excessive spreading of the elements. It also allows the use of a smaller angle of incidence $1 / 2 \theta$, thus permitting less foreshortening of the aperture due to vignetting of the beam-dividing element.

Light from source $\mathrm{S}$ is collimated by lens $\mathrm{L}_{1}$ (see fig. 1). A consideration of two rays of light, 1 and 2 , permits a general description of the instrument. Ray 1 is incident on the beam divider, $\mathrm{B}$, at $\mathrm{P}$ where it divides into two equal components $1_{T}$ and $1_{R}$; component $1_{T}$, taking the counterclockwise course, is transmitted by $\mathrm{B}$ to mirrors $\mathrm{M}_{1}, \mathrm{M}_{2}, \mathrm{M}_{3}$, and returning to $\mathrm{B}$ at point $\mathrm{P}^{\prime}$. The other component, $1_{R}$, is reflected by $\mathrm{B}$ at $\mathrm{P}$, traveling a clockwise course from $\mathrm{B}$ to $\mathrm{M}_{3}, \mathrm{M}_{2}, \mathrm{M}_{1}$ and returning to $\mathrm{B}$ at $\mathrm{P}^{\prime}$. After recombination at $\mathrm{P}^{\prime}$ the two components of ray 1 are focused by lens $\mathrm{L}_{2}$ at $\mathrm{E}$ where it is received either by the eye for visual observation or by a camera lens for photographic recording. Ray 2 is divided at $\mathrm{P}^{\prime}$ into two equal components $2_{T}$ and

${ }_{1}^{1}$ C. Candler, Modern interferometers, p. 209 (Hilger \& Watts, Ltd., London, 1951).
$2_{R}$. Ray $2_{T}$ travels the same route as $1_{R}$ but in the opposite direction, whereas ray $2_{R}$ travels the same route as $1_{T}$ but also in the opposite direction. Rays $2_{T}$, and $2_{R}$ recombine at $P$. One half of each beam is focused by lens $L_{2}$ at $E$ and the other half returning to the source $\mathrm{S}$. If the air through which the beams travel is assumed to be optically homogeneous, the two components of ray 1, although traveling different paths from $\mathrm{P}$ to $\mathrm{P}^{\prime}$, may be made to travel equal optical paths. The order of interference over the entire field will then be constant and quite near zero. If the beam-dividing surface is a dielectric, this constant is presumed to be zero; if metallic, it may differ from zero by a small quantity that varies with the angle of incidence, $\theta$. However, as the differential phase-shift at the beam-dividing surface does not enter into the working equations of this interferometer, the order of interference will be assumed to be proportional to the optical-path difference between the two component beams that recombine to produce interference.

If the beam divider, or either of the three mirrors, is rotated slightly about an axis through $\mathrm{P}_{0}$ (the center of the field) and perpendicular to the plane of the drawing, fringes will appear parallel to this axis of rotation. The order of interference increases on one side of $\mathrm{P}_{0}$, decreases on the other side, and remains zero at the center of the field. A dark fringe, that remains vertical or perpendicular to the plane of incidence, persists at the center. Vibrations which cause relative rotations of the interferometer elements cause only horizontal movements of fringes. The center of the central fringe appears fixed, regardless of vibrations.

The interferometer elements must be distributed so as to provide ample working space for large specimens that are to be located near a point in the path where the two wavefronts of a divided beam meet in their transit around the light circuit. This point will be half way around the circuit from the beam-dividing surface. The central plane of the specimen, which bisects the angle formed by its faces, is to be placed nearly normal to the divided beam.

Mirrors $\mathrm{M}_{2}$ and $\mathrm{M}_{3}$ are mounted to form an approximate right angle with each other and bound together into a rigid unit. The 10 -in. mirrors, used here, were mounted on separate tripods and the two tripods placed on a thick sheet of iron that could be moved about the table as a unit. All other elements could be moved independently. 


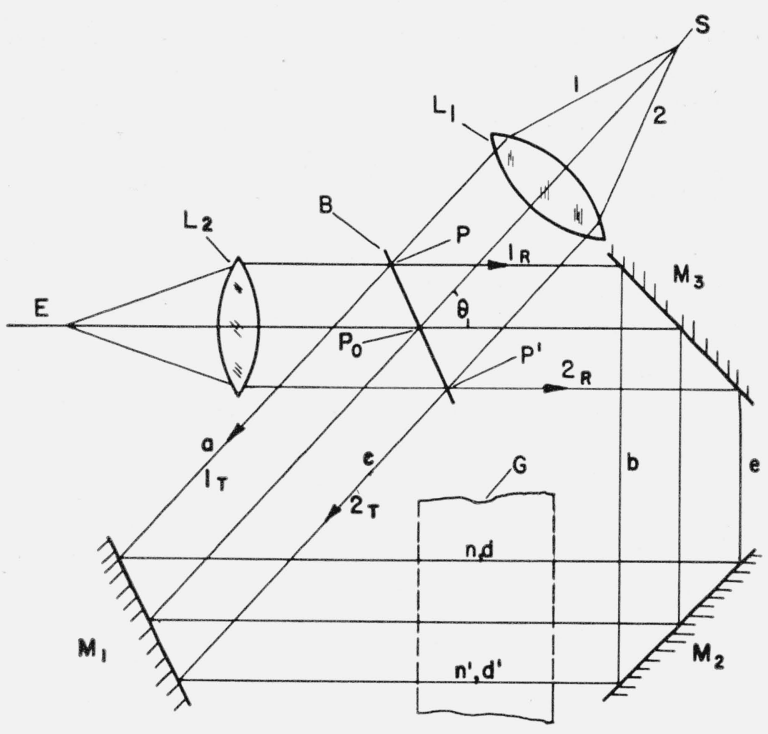

Figure 1. Modified Dowell Gage interferometer

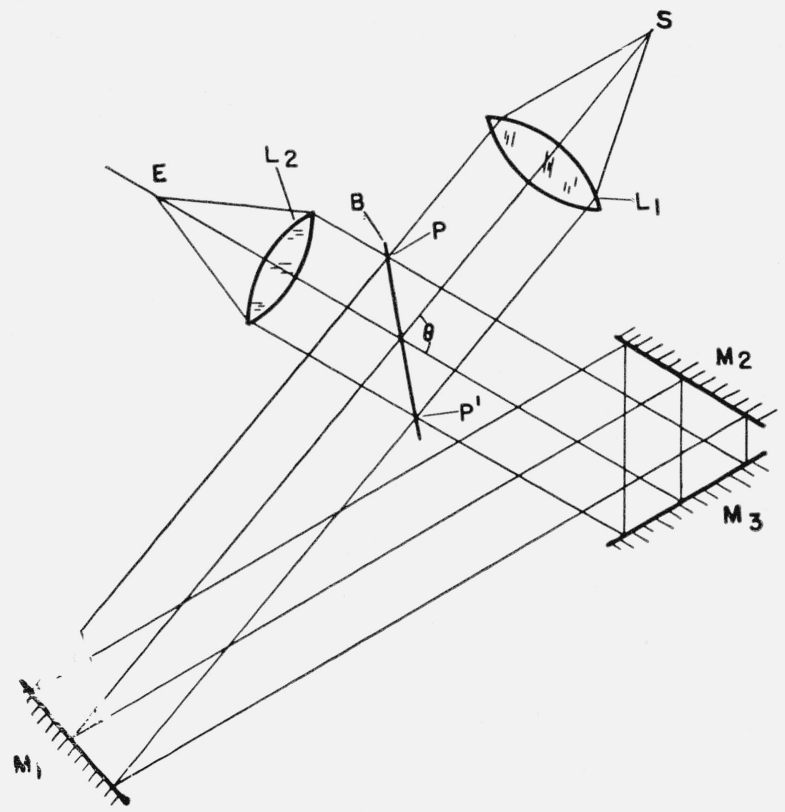

Figure 2. Dowell Gage interferometer.

If the apertures of all elements are assumed to be equal and circular, the maximum working aperture is obtained with a minimum value for $\theta$, consistent with required working space. If $\theta$ is chosen too small, the mirrors will require excessive spreading. A good compromise between angle of incidence and working-space requirements is indicated approximately by the drawing of figure 1 . The alinement of the elements to obtain maximum working aperture, for the chosen angle of incidence, $\theta$, is attained by placing a bright source of light at $\mathrm{S}$, with lens $\mathrm{L}_{1}$ and the beam divider $\mathrm{B}$ in place. The beam divider is placed symmetrically with the circular beam that is transmitted by lens $\mathrm{L}_{1}$ so that symmetrical vignetting is obtained. Mirror $\mathrm{M}_{1}$ is placed to receive the reduced beam centrally and normally. A part of the beam will then return into the source, which may now be more accurately adjusted to the focus of the lens. The axis of the lens is adjusted to pass through the center of the source. Mirror $\mathrm{M}_{3}$, forming a rigid unit with $\mathrm{M}_{2}$ as described above, is placed to receive the light reflected from $\mathrm{B}$, centrally and approximately $45^{\circ}$ to its normal. If mirror $\mathrm{M}_{2}$ is improperly placed, relative to $\mathrm{M}_{3}$, it may be corrected at this stage of adjustments. It will then receive the beam from $\mathrm{M}_{3}$ centrally and approximately $45^{\circ}$ to its normal. The essential operation in the adjustments of the mirrors is to return the two components of any divided ray to a common point on $B$ for recombination and in such manner that the two beams shall be coincident. This will be accomplished if the two components of the axial ray are: (a) caused to intersect at $\mathrm{M}_{1}$ by adjusting the direction of the reflected component by means of $\mathrm{M}_{2}$, (b) by placing $\mathrm{M}_{1}$ to receive these two components at the center of its face, and (c) by adjusting the orientation of $\mathrm{M}_{1}$ so that the normal at its center bisects the angle formed by the two incident components of the axial ray. These adjustments are facilitated by placing a pointed object, such as an arrow or lead pencil, close to lens $L_{1}$, with its point on the optic axis. The pencil projects a shadow along the two collimated beams. The points of the two shadows, one formed by the reflected component and the other by the transmitted component of the beam, will coincide at all points. The degree of coincidence is easily observed by placing a translucent screen (a thin sheet of white paper will do) to receive the two beams. This completes the alinement and preliminary adjustments of the elements. Further fine adjustments, to obtain satisfactory interference fringes, are most conveniently made at $B$.

It will be seen that the components of a ray that divides at $\mathrm{P}^{\prime}$ travel the same path as those traveled by the components of a ray that divided at $\mathrm{P}$, but in opposite directions, and recombine at $\mathrm{P}$. There is a relationship between these two points that does not exist between either of them and any other point. If a visible object is placed at any point in the path of either divided component of a ray from $\mathrm{P}$ to $\mathrm{P}^{\prime}$ two images of it are observed, one by light that recombines at $\mathrm{P}$ and the other by light that recombines at $\mathrm{P}^{\prime}$. We will, therefore, define $\mathrm{P}$ as the conjugate of $\mathrm{P}^{\prime}$ and vice versa. The order of interference, produced by the above-mentioned beams of light, at these two points are always equal in magnitude but opposite in sign. The interference pattern is, therefore, symmetrical about a vertical line through its center, one side of the field appears as a mirror image of the other, both with respect to interference fringes, markings on the surfaces of the plates, and objects in the path of the light (see fig. 3).

If a sample of glass or other transparent material with surfaces that are approximately plane and parallel is inserted in the beam at nearly normal incidence, the fringe pattern will be altered. The change 


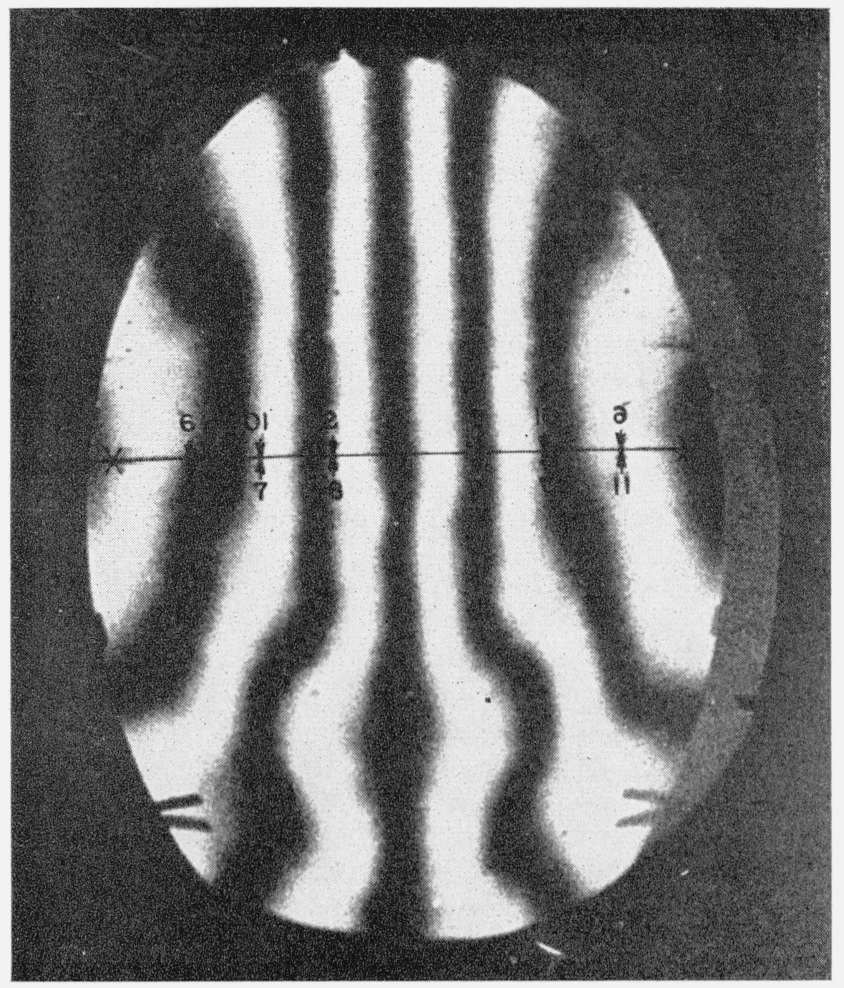

Figure 3. Transmission fringes.

All markings on one side of the field are imaged also on the other side.

in order at $\mathrm{P}$ will be equal to the change at $\mathrm{P}^{\prime}$ but of opposite sign. The change in the path cifferences for the component rays through points $\mathrm{P}$ and $\mathrm{P}^{\prime}$ correspond to the observed change in the number of fringes between $\mathrm{P}$ and $\mathrm{P}^{\prime}$. If the specimen is of uniform thickness, $d$, over the entire field this change in order of interference is a measure of the difference, $\Delta n$, in the average index through the plate along the two paths. If the difference in thickness of the plate is $\Delta d$, for the two paths, the observed change in fringe pattern is a function of both $\Delta d$ and $\Delta n$.

In general, both $\Delta d$ and $\Delta n$ are appreciable. We, therefore, need two independent relationships between $\Delta d, \Delta n$, and the observed orders of interference in order to compute values for either. A second relationship is found as follows: It may be noted that each of the two components of rays 1 and 2 suffer partial reflection, both externally and internally, at the two surfaces of sample $G$, shown in figure 1. If these surfaces are normal to the beams, these reflected component rays will return to their respective division points, $\mathrm{P}$ and $\mathrm{P}^{\prime}$. Four reflected beams, therefore, return to $\mathrm{P}$, two resulting from internal reflection in $\mathrm{G}$ and two from external reflection from G. Regardless of the thickness of $G$ the externally reflected rays may be made to travel equal paths that result in zero orcer of interference. This may be accomplished by moving $G$ parallel to the incident beams, which increases the path of one reflected component and decreases the other by

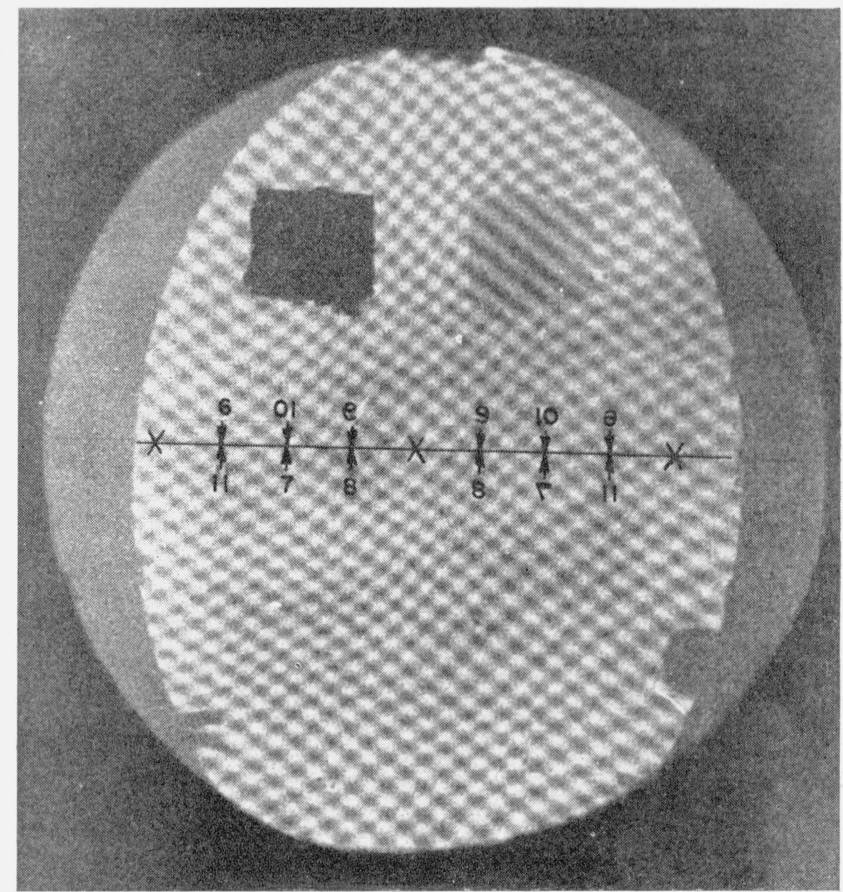

Figure 4. Reflection fringes.

an equal amount. If $\Delta d$ and $\Delta n$ are small, each of the two pairs of beams may be adjusted to produce interference of low order. The same is true for the corresponding beams that return to $\mathrm{P}^{\prime}$, after partial reflections internally and externally from $\mathrm{G}$. These two sets of fringes are inseparable (see fig. 4). They may, however, be separated from the fringes formed by the two transmitted beams that converge to $\mathrm{E}$ by rotating the sample $\mathrm{G}$ until the two overlapping images of the source, formed by light reflected by G and imaged also in the focal plane of $\mathrm{L}_{2}$, are sufficiently separated from E to permit screening them from the brighter beam focused at $\mathrm{E}$. It may be shown that a small rotation of $G$ does not affect the differences in order of interference between $\mathrm{P}$ and $\mathrm{P}^{\prime}$ because the deviation is equal for all beams reflected from G. A single set of reflection fringes is desired, but as the two sets are inseparable, both must be accepted.

\section{Definition of Fringes}

We have discussed the origin of four sets of fringes. These will be defined as follows: (a) The "openinterferometer fringes" observed form E with no specimen in the beam, (b) the "transmission fringes", so designated because they are produced by light transmitted through the sample, (c) the "externally reflected fringe" produced by light reflected externally by the sample, and (d) the "internally reflected fringes" produced by light reflected internally in the sample. To these will be added another set that enters into the discussions. They are (e) the "white-light fringes" formed from an extended source located near the beam divider. 


\subsection{White-Light Fringes}

After the elements of the interferometer are adjusted according to the above instructions, a small light source, such as a glowing flashlight bulb, is placed on the axis of lens $L_{1}$ or $L_{2}$ and near the incident face of the beam divided. On looking through the optical train from the side of the beam divider opposite the source, one sees two images of this light. By adjusting the beam divider one may control the separation of the two images of the light. By this means they are made to appear superimposed on each other. A small telescope located in the position shown for $L_{2}$ in figure 1 permits closer superposition of these images. One of these images is formed by the transmitted component of the light beam and the other by the reflected component. After the two images are superimposed, white-light fringes should be visible when the flashlight is replaced with an illuminated screen and the telescope is replaced by the eye of the observer. Both eyes are used if the aperture exceeds their separation. Room illumination is usually sufficient for illuminating this screen. The screen and the observer must be far enough from the beam divider to avoid obstruction of light between the beam divider and the mirrors. On looking through the obtical train one sees the white-light fringes, usually ine, and at some arbitrary orientation. Further fine adjustments of the beam divider, while observing these fringes, permit the operator to adjust these fringes, so that one fringe covers the entire aperture.

Best contrast is obtained in the interference fringes that are used for making measurements, if the white-light fringes are parallel to the plane of figure 1 .

\subsection{Open-Interferometer Fringes}

After the above adjustments the white screen is removed and, with lens $\mathrm{L}_{2}$ in place, the eye located at $\mathrm{E}$ will observe a second set of white-light fringes that are produced by a white source located at $\mathrm{S}$. These fringes are vertical with the black or zeroorder fringe at the center. The separation of these fringes can be altered by rotating $B$ about an axis that is normal to the plane of figure 1 . The configuration is symmetrical about the central black fringe.

\subsection{Transmission Fringes}

The open interferometer fringes of 3.2 above become the transmission fringes when the sample is inserted into the beam. If the sample is smaller than the beam, or only intercepts a part of it, both sets are seen simultaneously in adjacent parts of the field. The differences in the configuration of the open interferometer and transmission fringes are caused by changes in the optical path resulting either from variations in index of the sample, variations in geometrical thickness of the sample, or both. In order to isolate these two quantities the reflection fringes must be considered.

\subsection{Reflection Fringes}

The origin of the reflection fringes have been discussed above. If low-order interference is to be obtained the paths traveled by the two components of each reflected beam must be nearly equal. The adjustments for this are as follows: The position for the central plane of the sample is properly located by placing an object or light source between mirrors $\mathrm{M}_{1}$ and $\mathrm{M}_{2}$ and observing the two images of it with both eyes from a position between $\mathrm{B}$ and one of the lenses. The correct position for the center of the specimen is half way between the two observed images. Its exact position is obtained by moving this object or light source along the beam until the two observed images appear coincident or equally distant from the observer. If the aperture is too small to use both eyes for stereo perception of distances, a telescope may be used as a range finder by focusing alternately on the two images.

The center of the specimen is to be placed where the light is located and adjusted normal to the beam by observing the four images formed by light reflected from the specimen and located in the focal plane of $\mathrm{L}_{2}$. If the angle between the two surfaces of the sample is small enough for this test to be applied, these images will overlap in pairs and two separated double images will be observed. There will be other images, much fainter than the ones mentioned here, but these are due to multiple reflections in the specimen and also reflections from the unsilvered surface of the beam divider. These are easily identified and need no further consideration.

The width, or separation, of the reflection fringes are controlled by rotating $G$ about an axis that is parallel both to its surfaces and to the plane of figure 1. Contrast will be poor if the specimen is not correctly located. If contrast is poor, it may be improved by moving $\mathrm{G}$ along the beam so as to make the path difference between the component beams equal.

\section{Symbols and Abbreviations}

The derivation of the working formulas for this interferometer requires a considerable number of symbols. The definitions of these follow: (see fig. 1). The quantities $a, b, c$, and $e$ represent the optical paths traveled by $1_{\mathrm{T}}, 1_{\mathrm{R}}, 2_{\mathrm{T}}$, and $2_{\mathrm{R}}$, respectively, from $\mathrm{B}$ to $\mathrm{G} ; d$ and $d^{\prime}$ represent the geometrical paths traveled in the specimen by rays reflected from $\mathrm{P}$ and $\mathrm{P}^{\prime}$, respectively; $n$ and $n^{\prime}$ are the indices of 1 efraction along paths $d$ and $d^{\prime}$, respectively; $F_{\nu}$ and $F_{\nu}^{\prime}$ are the orders of interference observed at $\mathrm{P}^{\prime}$ and $\mathrm{P}^{\prime}$, respectively, where $\nu=1$ for the open interferometer fringes, $\nu=2$ for the transmission fringes, $\nu=3$ for the externally reflected fringes and $\nu=4$ for the internally reflected fringes $; F_{\nu}-F_{\nu}{ }^{\prime}=\Delta F_{\nu} ; d-d^{\prime}=\Delta d$; $n-n^{\prime}=\Delta n$; and $\lambda$ is the wavelength of the spectrally homogeneous light used. 


\section{Derivation of Formulas}

In order to apply algebraic operations to the derivation of an equation relating $\Delta n$ to known quantities, we must establish a convention for signs. We will, accordingly, define the direction of increasing order of interference at $\mathrm{P}$ to correspond to the change obtained by a clockwise rotation of the beam divider about a vertical line (perpendicular to the plane of fig. 1) through the center of its surface. That is, a decrease in either $b$ or $c$ increases the order at $\mathrm{P}$ and decreases it at $\mathrm{P}^{\prime}$. Likewise, an increase in either $a$ or $e$ increases the order at $\mathrm{P}^{\prime}$ and decreases it at $\mathrm{P}^{\prime}$. With this convention and the above definitions, it is seen from figure 1 that

$$
\begin{aligned}
F_{1} \lambda & =(a+d+e)-\left(b+d^{\prime}+c\right), \\
F_{1}^{\prime} \lambda & =\left(c+d^{\prime}+b\right)-(e+d+a)=-F_{1} \lambda, \\
\therefore \Delta F_{1} & =\left(F_{1}-F_{1}^{\prime}\right)=2 F_{1} .
\end{aligned}
$$

Similarly,

$$
\begin{aligned}
& F_{2} \lambda=(a+n d+e)-\left(b+n^{\prime} d^{\prime}+c\right), \\
& F_{2}^{\prime}=-F_{2},
\end{aligned}
$$

or

$$
\begin{aligned}
\Delta F_{2} & =\left(F_{2}-F_{2}^{\prime}\right)=2 F_{2} . \\
F_{3} \lambda & =2(a-b), \\
F_{3}^{\prime} \lambda & =2(c-e),
\end{aligned}
$$

or

$$
\begin{aligned}
\Delta F_{3} \lambda & =\left(F_{3}-F_{3}^{\prime}\right) \lambda=2(a+e)-2(b+c) . \\
F_{4} \lambda & =2(a+n d)-2\left(b+n^{\prime} d^{\prime}\right), \\
F_{4}^{\prime} \lambda & =2\left(c+n^{\prime} d^{\prime}\right)-2(e+n d),
\end{aligned}
$$

or

$$
\Delta F_{4} \lambda=2(a+e+2 n d)-2\left(b+c+2 n^{\prime} d^{\prime}\right) .
$$

Since, by definition, $n^{\prime}=n-\Delta n$ and $d^{\prime}=d-\Delta d$, $n^{\prime} d^{\prime}=(n-\Delta n) \quad(d-\Delta d)=n d-n \Delta d-d \Delta n, \quad$ approximately. Therefore,

and

$$
\begin{gathered}
n d-n^{\prime} d^{\prime}=n \Delta d+d \Delta n \\
F_{1} \lambda=a+e-b-c+\Delta d \\
F_{2} \lambda=a+e-b-c+(n \Delta d+d \Delta n)
\end{gathered}
$$

On eliminating the $F \nu$ 's from eq (1), (3), (7), and (8), we get

$$
\begin{gathered}
\Delta F_{1} \lambda=2(a+e-b-c+\Delta d) \\
\Delta F_{2} \lambda=2(a+e-b-c+n \Delta d+d \Delta n) .
\end{gathered}
$$

Equations (4), (9), (10), and (11) form a set of four equations with the three unknown quantities
$\Delta d, \Delta n$, and $(a+e-b-c)$. Three equations selected from this set are sufficient to evaluate $\Delta n$ if the specimen can be inserted into or withdrawn from the beam without disturbing the relative positions of the interferometer elements. In order to read the reflection fringes the specimen must be rigidly attached and adjusted to the interferometer in order to avoid instability in the fringes during the recording. This may be affected with a small interferometer, but it was considered that forces necessary to hold a large specimen would probably deform the table on which the elements rested. The large specimen, however, does not have to be bound rigidly to the interferometer for reading the transmission fringes because small rotations and displacements produce negligible effects on the transmission fringes. Consequently, the term $(a+e-b-c)$ may be held constant between readings of the open interferometer and transmission fringes and, therefore, eliminated from eq (10) and (11), giving the equation

$$
\left(\Delta F_{2}-\Delta F_{1}\right)=2(n \Delta d+d \Delta n-\Delta d) .
$$

The term $(a+e-b-c)$, although differing slightly in value between the time of reading the transmission and reflection fringes, remains constant between readings of the two sets of reflection fringes. It, therefore, may be eliminated from eq (4) and (9), giving the relation

$$
\left(\Delta F_{4}-\Delta F_{3}\right)=4(n \Delta d+d \Delta n) .
$$

On eliminating $\Delta d$ from (12) and (13) and solving for $\Delta n$, we have

$$
\Delta n=\frac{\lambda}{4 d}\left[2 n\left(\Delta F_{2}-\Delta F_{1}\right)+(n-1)\left(\Delta F_{3}-\Delta F_{4}\right)\right] .
$$

\section{Determination of Algebraic Signs}

If a light, steady pressure is applied to one side of the cell that holds the beam divider $\mathrm{B}$, and in a direction that tends to rotate it clockwise, the path length $(a+e)$ increases and the path length $(b+c)$ decreases. That is, $F_{\nu}$ increases and $F_{\nu}^{\prime}$ decreases. Consequently, $\Delta F_{\nu}(\nu=1,2,3$, or 4) increases algebraically according to the accepted convention when this test is performed. These quantities are, therefore, positive or negative, depending upon whether the rotational operation increases or decreases the quantity in absolute magnitude. This permits the determination of sign to be applied to the observed $\Delta F_{\nu}$ 's for insertion in formula (14), and consequently the direction and magnitude of the change in index between any two conjugate points on the specimen.

\section{Identification of Fringes}

The identification of the $F_{1}$ (open interferometer) fringes and the $F_{2}$ (transmission) fringes are obvious. The $F_{3}$ (external reflection) fringes and the $F_{4}$ (internal reflection) fringes move quite vigorously 
for small movements of the specimen. They move together, however, and are not distinctively identified one from the other without further tests.

If a small piece of black paper (shown in fig. 4) that is coated with petroleum jelly or other material having an index of refraction closely approximating that of the glass is applied to one surface of the specimen and in the light beam but away from the chosen fiduciary reference points, it eliminates both internal and external reflections from the area covered. It also eliminates internal reflection from that part of the other surface of the specimen, opposite the coated area, where the paper obstructs the beam. Consequently, the $F_{3}$ fringes will appear in only one of the two conjugate images of the paper, and the $F_{4}$ fringes will appear in neither image. Identification is thus effected.

\section{Choice of Reference Points}

The choice of reference points depends upon the nature and extent of the data wanted. If the required information is limited to the average gradient between two chosen points and the separation is less than or equal to the horizontal diameter of the interferometer aperture, then the specimen is placed so that the two chosen points are conjugate to each other. If the separation of the two points exceeds the aperture, then the total index variation must be measured in steps. Intermediate points are chosen that may be made conjugate in pairs, and data are taken for the differences between all adjacent points. The algebraic sum of these differences equals the difference in index for the extreme points.

If the variation in index gradient between two remote points on the specimen is desired, then a larger number of reference points are chosen between the two limiting ones. If the reference points are chosen equally spaced and on a straight line (or on the arc of a circle), any number of points may be used with relatively few shifts in position of the specimen. To illustrate this procedure, we will choose the spacing between the points to be slightly less than one-third the horizontal diameter of the interferometer aperture (see fig. 5). That is, points $1,2,3$, and 4 may be adjusted in the beam so that point 1 is conjugate to 4 and point 2 conjugate to 3. Figure $5, \mathrm{~A}$, shows the reference points and the observed mirror images. With the specimen in position $\mathrm{A}^{\prime}$, values are found for $\Delta n_{1,4}$ (the difference in index between points 1 and 4) and $\Delta n_{2.3}$. The specimen is then shifted to a new position $\mathrm{B}^{\prime}$ (fig. $5, \mathrm{~B}$ ) such that point 3 becomes conjugate to 6 and point 4 conjugate to 5 . Figure $5, \mathrm{~B}$, shows the apparent position of reference points. Values for $\Delta n_{3,6}$ and $\Delta n_{4,5}$ are then obtained. 'The specimen is then shifted to position $\mathrm{C}^{\prime}$, where point 6 becomes conjugate to 7 and 5 to 8 . This procedure is continued until all points are covered. We then have the following data: $\Delta n_{1,4}$, observed directly; $\Delta n_{1,5}=\Delta n_{1,4}+\Delta n_{4,5} ; \quad \Delta n_{1,8}=\Delta n_{1,5}+\Delta n_{5,8} ; \quad$ etc., $\Delta n_{2,3}$

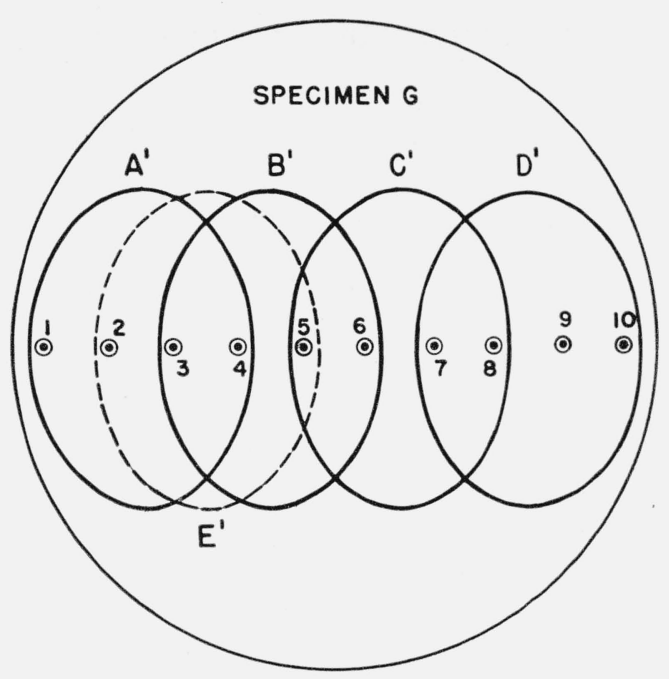

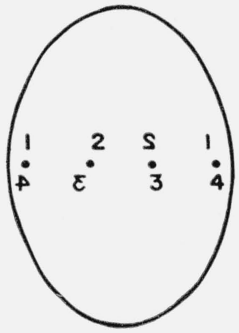

A

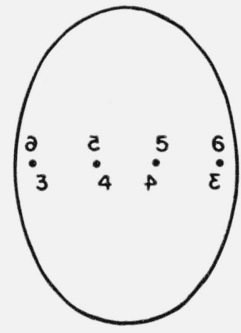

B

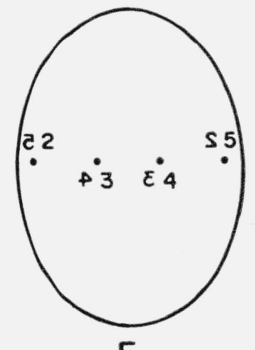

$\mathrm{E}$
FIGURE 5. Distribution of reference points on the sample, $G$, relative to the beams for several settings.

The insets $\mathrm{A}, \mathrm{B}$, and $\mathrm{E}$ represent the observed reference points and their associated numbers, as seen when the beam is located at $A^{\prime}, B^{\prime}$, and $\mathrm{E}^{\prime}$, respectively.

observed directly; $\Delta n_{2,6}=\Delta n_{2,3}+\Delta n_{3,6}$, etc. These values represent the index differences between any two points of the group $1,4,5,8$, etc., and similarly for group $2,3,6,7$, etc. The data for either group is sufficient to determine the variation in index gradient along the line of reference points. A curve relating $n$ to the position coordinates of the points may be obtained for either set of points if $n$ is known for one point in each group. The two curves may be superimposed upon each other by adding a constant (determined statistically or otherwise) to all $n$-values of one set or an additional setting may be made to determine the difference experimentally. By placing the specimen so that reference point 2 becomes conjugate to 5 and 3 conjugate to 4 (position $\mathrm{E}^{\prime}$, fig. 5) we may obtain $\Delta n_{2,5}$ and $\Delta n_{3,4}$. These values, together with those already obtained, permit a determination of index differences between any tow of the chosen reference points. If more points are desired, they may be inserted between the above specified points and other sets of data are obtainable for more detailed values of index gradients. 


\section{Reflectivity of Surfaces}

In order to obtain good contrast it is essential that the two component beams that produce interference be of equal flux. In this interferometer the reflectivity-transmissivity ratio of the beam-dividing face does not affect the contrast of the two sets of reflection fringes because the beams are affected equally, one reflection and one transmission being suffered by each. Consequently, because the reflection fringes are faint relative to the other fringes, maximum flux density is desired and obtained in these by making the reflectance of the beam divider equal to its transmittance. Fortunately, this ratio is that required also for maximum contrast in all other sets of fringes. The reflections of the end mirrors have no effect on the other fringes due to equality of effects on all beams that combine to produce them. Thus, by making the reflectance of the beam divider equal to its transmittance, maximum contrast is obtained in the white-light, open-interferometer, and transmission fringes and maximum flux density in all sets of fringes. The fluxes of the component beams that form either set of reflection fringes will, however, be unequal if the reflectance of the three end mirrors are made a maximum as is done for most other types of interferometers. These should be equalized by reducing the fluxes of the stronger components to that of the faintest. Thus, if the maximum reflectance obtainable is 90 percent, mirrors $\mathrm{M}_{2}$ and $\mathrm{M}_{3}$ should be given this maximum reflectance, and mirror 1 should be given a reflectance of 81 percent (i. e., $0.90^{2}$ ). This will reduce the flux density of all other fringes proportionally, but they are so much brighter than the reflection fringes that no real harm is done.

\section{Conclusions}

This interferometer permits accurate measurements of lateral variation of refractive index in transparent bodies of almost any thickness. It has been used quite successfully to measure index variations in glass blocks up to 5 in. thick and disks up to $27 \mathrm{in}$. in diameter. This interferometer does not require the use of narrow spectral lines such as is required for observing high-order interference, but any spectrally homogeneous (monochromatic) light source is usable.

Washington, May 11, 1954. 